\title{
Study protocol for a multicentre, randomised controlled trial of the external use of mirabilite to prevent pancreatitis in children after endoscopic retrograde cholangiopancreatography.
}

\section{Zhaohui Deng}

Shanghai Jiao Tong University School of Medicine https://orcid.org/0000-0001-6975-6453

Biao Gong ( $\sim$ gongbiaoercp@163.com )

Shanghai University of Traditional Chinese Medicine https://orcid.org/0000-0002-3645-2493

Kaihua Yang

Shanghai Jiao Tong University School of Medicine

Jingqing Zeng

Shanghai Jiao Tong University School of Medicine

Chan Lv

Shanghai Shuguang Hospital

Jianmei Ji

Shanghai Shuguang Hospital

\section{Xingjia Li}

Shanghai Shuguang Hospital

\section{Zhijian Gu}

Shanghai Shuguang Hospital

\section{Weituo Zhang}

Shanghai Jiao Tong University School of Medicine

\section{Study protocol}

Keywords: Mirabilite, Post-ERCP pancreatitis, Protocol, Children

Posted Date: January 13th, 2021

DOI: https://doi.org/10.21203/rs.3.rs-20402/v1

License: (c) (1) This work is licensed under a Creative Commons Attribution 4.0 International License. Read Full License 


\section{Abstract \\ Background}

Acute pancreatitis is the most common complication of endoscopic retrograde cholangiopancreatography (ERCP) in children. To date, there is no suitable medicine for post-ERCP pancreatitis prophylaxis in children and few study has prospectively evaluated an intervention to prevent post-ERCP pancreatitis in children. Mirabilite, a well-known traditional Chinese medicine(TCM) has good therapeutic effects on acute pancreatitis and no side effect for children by basic research and clinical studies. Our protocol is designed to assess the efficacy and safety of the external use of mirabilite to prevent post-ERCP pancreatitis in children.

\section{Methods/design:}

520 patients planned for diagnostic and therapeutic ERCP will be enrolled according to the eligibility criteria. The patients will be randomly divided into two equal groups (Mirabilite and control, the external use of mirabilite in a bag on the projected abdominal area over the pancreas within 30 min before ERCP). The primary end point is incidence of post-ERCP pancreatitis. Secondary end points include abdominal pain scores, the levels of inflammatory markers [tissue necrosis factor (TNF)-a, IL-6, and IL-8] and intestinal barrier function markers (diamine oxidase, D-lactic acid, and endotoxin). Additionally, the side effects of topical mirabilite is investigated.

\section{Conclusion}

This trial would be the first experiment to determine mirabilite to prevent post-ERCP pancreatitis in children. Mirabilite maybe provide potential clinical benefits and a new avenue with tremendous potential for the future preventing of post-ERCP pancreatitis.

\section{Trial registration number:}

ChiCTR1900022642. Registered on 19 April 2019- Retrospectively registered, http://www.chictr.org.cn.

\section{Background}

Endoscopic retrograde cholangiopancreatography (ERCP) is an advanced endoscopic technique that is well established for diagnosis and treatment in biliary and pancreatic disorders in children ${ }^{1,2}$. Post-ERCP pancreatitis is the most common adverse event following ERCP occurring in approximately $6-10.9 \%$ of children varying between case series dependent on multiple factors, including case mix, operator characteristics, underlying disease factors and variations in post-ERCP management ${ }^{3-5}$. Most episodes 
of post-ERCP pancreatitis are mild but a small proportion of patients develop severe pancreatitis, leading to prolongation of hospital stay, a long stay in the Intensive Care Unit that can increase the morbidity and mortality rates. The occurrence of post-ERCP pancreatitis limits the extensive application of ERCP in children.

The pathophysiology of post-ERCP pancreatitis is not entirely clear with several factors such as chemical, mechanical or microbiological factors that result from papillary instrumentation and/or hydrostatic injury from the overfilling of the pancreatic duct with contrast material ${ }^{6,7}$. The influence of these factors leads to activation of trypsinogen, oxidative stress, ischemia, at last inducing tissue injury. Activating inflammatory reaction chain and transcription of pro-inflammatory cytokines play an important role in tissue injury. It can induce chemo-attraction of mono-macrophages which increases the pro-inflammatory cascade and increase tissue necrosis.

To date, only non-steroidal anti-inflammatory drugs (NSAIDs) have been shown as effective in the prevention of post-ERCP pancreatitis in adult ${ }^{8,9}$. Questions remain regarding the dose of rectal indomethacin widely used in the adult population is not utilized in children and the method of rectal administration is not acceptable for children. Furthermore, hardly any reports have researched prophylaxis medicine for post-ERCP pancreatitis in children. Finding an ideal, effective, less-invasive and safe prevention for children are desired.

Mirabilite, a white granular mineral medicine primarily hydrous sodium sulfate $(\mathrm{Na} 2 \mathrm{SO} 4 \mathrm{10H} 2 \mathrm{O})$ has been a well-known traditional Chinese medicine (TCM) for treating acute pancreatitis. Under the guidance of TCM pharmaceutical theory, acute pancreatitis is categorized as epigastric pain, splenic precordial pain, splenopyretic disease and knotted chest disease. The principle of treatment in mirabilite is to clear away the heat-evil (heat as a pathogenic factor that causes heat pattern/syndrome) and expelling superficial evils, supplementing qi (vital energy) and nourishing yin (body fluid), activating blood circulation to dissipate blood stasis and inner communication and purgation. Many studies have shown that mirabilite plays an important role in the treatment of acute pancreatitis and other inflammatory diseases in adult in china. ${ }^{10-12}$. Li et al ${ }^{13}$ applied mirabilite to reduce pancreatic leakage of severe acute pancreatitis, which can lower intra-abdominal pressure, reduce the secretion of pancreatic amylase, eliminate inflammatory edema, and reduce IL- 6 levels in the blood. Wang et al ${ }^{14}$ found that mirabilite promoted the recovery of intestinal mucosal permeability and prevented of sepsis acute gastrointestinal injury in patients with severe sepsis. Animal experiments have shown that Dachengqi decoction, famous formulas in China which comprises mirabilite serving as the principal component increases cell viability, induces pancreatic acinar cell apoptosis, reduces acinar necrosis and protects from injuries to the pancreas in vivo and in vitro ${ }^{15,16}$. The likely mechanisms may operate through reducing ROS generation and regulating the nitric oxide pathway in rat with acute pancreatitis. Although there is no studies about mirabilite treating for acute pancreatitis in children, external use of mirabilite has been proved to be effective and safe for treating acute suppurative appendicitis, pneumonia, tonsillitis of children in china ${ }^{17,18}$. 


\section{Research Hypothesis}

Basing on mirabilite can treat acute pancreatitis, we hypothesis that mirabilite is effective in preventing post-ERCP pancreatitis. Furthermore, previous clinical evidences have shown that mirabilite is a safe and beneficial treatment option for children with inflammatory diseases.

\section{Trial design}

In this study, mirabilite is applied for preventing Post-ERCP pancreatitis in children and the efficacy and safety of the external use of mirabilite is assessed by a the medical staff and patient blinded, multicentre, controlled, observe, randomised with a 1:1 allocation trial.

\section{Methods/design}

Study design In this research, clinical trial will be performed in Shanghai Children's Medical Center and Shanghai Shuguang Hospital in China. A brief flowchart of the entire study is shown in Fig.1, and the schedule of events is provided in Fig. 2.

\section{Objectives}

1. The primary research objective is to evaluate the efficacy and safety of the external use of mirabilite to prevent post-ERCP pancreatitis in children.

2. The secondary research objectives include to (a) assess the efficacy of mirabilite to relieve abdominal pain in children after ERCP, (b) the effect of mirabilite on intestinal barrier function and (c) pro-inflammatory reaction.

\section{Study populations}

Patients who planned to undergo ERCP in the participated hospitals will be invited to the study. A screening session and physical examination prior to inclusion will be conducted by a medical doctor according to the following criteria.

An investigator who is masked to treatment allocation records the patient demographics, post-ERCP adverse events and follow-up data and the procedure-related parameters including cannulation methods, numbers of cannulation attempts, and inadvertent pancreatic duct cannulation, pancreatography, and prophylactic placement of pancreatic duct stent.

\section{Inclusion criteria}

1. Ages: 0-14 years.

2. Planned for diagnostic or therapeutic ERCP. 
3. Blood pancreatin level before ERCP within normal limits (amylase: 30-110u/l, blood lipase: 23$300 u / l)$.

4. Informed consent obtaining from the guardians of all patients, and assent obtaining from patients greater than 10 years of age.

\section{Exclusion criteria}

1. Organic gastrointestinal disease such as upper digestive tract stenosis or obstruction.

2. Pancreatitis or use of pancreatic enzyme medication within 7 days.

3. Cardiovascular, hepatic, renal, cerebrovascular disease or hematopoietic system disease.

4. Dermatological disorders such as fresh abdominal wounds, skin lesions, or angioma. Allergy to contrast agents or mirabilite.

\section{Intervention}

Surgical details All pediatric patients undergo a comprehensive review and specialist consultations before ERCP. This process aims to ensure an objective and comprehensive analysis, as well as the appropriateness of ERCP, and to rule out contraindications to endoscopy. Patients are asked to undergo routine preoperative laboratory testing (complete blood count, coagulation, blood amylase and lipase concentrations, and hepatic function markers), upper abdominal ultrasonography, computed tomography (CT), magnetic resonance cholangiopancreatography (MRCP), and iodine allergy testing.

Each patient is required to fast for 12 hours before surgery. Duodenoscopy is performed using the JF240V devices (Olympus Corp, Tokyo, Japan). ERCP is conducted by an experienced digestive endoscopy specialist who had performed $>30000$ ERCPs. The following procedures are completed under radiographic guidance: endoscopic sphincterotomy, balloon dilation, stone extraction, and stenting and naso-pancreatic or naso-bilary drainage tube placement.

Standard post-ERCP treatment Standard treatment will be administered for post-ERCP pancreatitis of both groups, including fasting, pancreatic enzyme control, and fluid and electrolyte balance maintenance. Complications such as infection, bleeding, or perforation within 1 month after discharge will be treated accordingly.

Intervention group(External use of Mirabilite) $\bowtie$ Patients in the mirabilite group are administered topical application of mirabilite in bag on the middle and upper abdomen within 30 min before ERCP till $24 \mathrm{~h}$ after ERCP, during which time the mirabilite is replaced every 4 hours, while those in the control group do not receive any additional intervention. Mirabilite is provided by Shanghai University of Traditional Chinese Medicine. 
Mirabilite bag is designed with a rectangular shape and in two sizes based on the projected area of the pancreas of the pediatric patients. Children aged $\leq 6$ years receive bags with dimensions of $17 \mathrm{~mm} \times 14$ $\mathrm{mm}$, and those aged $>6$ years received bags with dimensions of $24 \mathrm{~mm} \times 14 \mathrm{~mm}$. Two layers of medical gauze are sewn into rectangular bags, and with four 8-cm attachment bands are sewn to the two longer sides. The bags are used for the topical application of mirabilite to the abdomen and are attached to the patient's back using the attachment bands.

\section{Randomisation and masking}

In this study, patients will be randomly assigned into two treatment groups by 1:1 ratio using a block randomization stratified by centres. The randomization is performed before ERCP (about 7h before ERCP, usually on the morning of the procedure).

Mirabilite will be administered in the procedure room before or after ERCP by one investigator in each site who do not participate in data collection and analysis. These investigators and patients will be instructed not to disclose if or when mirabilite is used. Endoscopists and assistances who participated in ERCP procedures will be masked to group allocation. Investigators who collected demographic or procedurerelated data or participated in the assessment of post-ERCP complications will be also masked from group allocation.

\section{Assessments and measurements}

Abdominal pain scores (visual analogue scale[VAS] scores) ${ }^{19}$ will be recorded for all patients at $24 \mathrm{~h}$ after surgery. On postoperative days 1, 2 and 3, TNF-a, IL-6, IL-8, diamine oxidase, blood D-lac, and endotoxin of serum will be measured. TNF-a, IL-6, IL-8 will be determined using the ELISA method (sandwich ELISA format). Diamine oxidase will be determined by spectrophotometry (Nanjing Jiancheng Bioengineering Institute, Nanjing, China). D-lac will be determined using modified enzymatic spectrophotometry (Nanjing Jiancheng Bioengineering Institute, Nanjing, China). The quantitative determination of endotoxin will be performed with a limulus kit (Sigma, St Louis, MO, USA).

According to the criteria diagnosis of post-ERCP pancreatitis, serum amylase levels and upper abdominal ultrasonography will be measured $24 \mathrm{~h}$ after ERCP.

\section{Outcomes}

\section{Primary outcome}

The primary outcome is the incidence of post-ERCP pancreatitis at $24 \mathrm{~h}$ after surgery. The post-ERCP pancreatitis (PEP) is defined if a children after ERCP met 2 of the 3 following criteria: classical abdominal pain, a plasma amylase or lipase concentration exceeding 3 times the normal upper limit at 24 hours postoperatively, and radiographic (B-type ultrasonography or CT) suggestive of pancreatitis. 
Secondary outcomes: Several secondary outcome measures are also recorded. Abdominal pain is measured $24 \mathrm{~h}$ after ERCP using a visual analogue scale (VAS), as follows: 0 points, no tenderness, no pain; 1-3 points, mild but tolerable discomfort and pain; 4-6 points, sleep quality affected by tolerable discomfort and pain; and 7-10 points, strong discomfort and intolerable pain that severely affects sleep quality. The intestinal barrier function assessed by diamine oxidase, D-lactic and endotoxin and proinflammatory cytokine assessed by concentrations of TNF- $a$, IL- 6 , and IL- 8 are recorded. Inpatient days, the rate of mortality and transfer to the ICU because of severe PEP are also recorded.Safety endpoints

Patients are monitored for adverse reactions to mirabilite, including skin damage and diarrhea. The following adverse reactions to ERCP are also monitored: post-surgical intestinal perforation, bleeding, bile duct infection, and other procedure-related complications requiring an extension of the hospital stay. Patients are followed up for 1 month postoperatively. Detailed definitions for other adverse events are provided in Table $1^{20}$.

Table 1: Consensus criteria for ERCP complications

\begin{tabular}{llll}
\hline & Mild & Moderate & Severe \\
\hline Bleeding & Clinical evidence of bleeding (i.e., & Transfusion: 4 units; no angiographic & Transfusion> 5 units or \\
& not just endoscopic); Hb level drop & intervention or surgery & or surgical) \\
& $<3 \mathrm{~g}$, and no need for transfusion & & Medical treatment for more \\
Perforation & Possible, or only very slight leak of & Any definite perforation treated & than 10 d, or intervention \\
& $\begin{array}{l}\text { fluid or contrast dye; treatable by } \\
\text { fluids and suction for } \leq 3 \text { days }\end{array}$ & medically for 4-10 d & (percutaneous or surgical \\
Infection & $>38^{\circ} \mathrm{C} 24-48 \mathrm{hr}$ & Febrile or septic illness requiring more & Septic shock or surgery \\
(cholangitis) & & than 3 d of hospital treatment or & endoscopic or percutaneous intervention
\end{tabular}

\section{Sample size estimation and statistical Analysis}

In our previous pilot study, the incidence of post-ERCP pancreatitis in control group was $26 \%$ and the incidence in mirabilite group was $16 \%$. The sample size was 260 subjects per group with a of 0.05 and statistical power of 0.8 .

We will report our results according to the CONSORT 2010 Statement. The data of all patients who underwent randomization will be analyzed. The qualitative variables will be presented as absolute numbers and proportions. Quantitative variables will be presented as mean \pm SD or medians and IQRs as appropriate. The primary endpoint, the incidence of PEP will be shown in absolute frequency and ORs with $95 \mathrm{Cl}$, and compared by $\chi^{2}$ tests. The secondary endpoints between the two groups will be compared by Mann Whitney $U$ test or t-test as appropriate. All tests are two-sided, and a p value of less than 0.05 is considered statistically significant. Subgroup analysis will be performed according to the patient demographics (age, sex, centre, et al) and the procedure-related parameters. Missing values will be 
imputed with multiple imputation under the assumption of missing at random. All statistical analyses will be performed with $\mathrm{R}$ (version 3.5.1).

\section{Discussion}

To our knowledge, our study is the first to evaluated prophylactic medications for pediatric post-ERCP pancreatitis inmulti-centre randomized controlled trial.

Mirabilite can reduce inflammation, promote the recovery of large intestinal function and the excretion of intestinal toxins, thus reducing damage to the intestinal mucosa. Mirabilite can also reduce symptoms such as abdominal pain, and may also decrease the risk of complications. Therefore, many clinical studies in China have evaluated the use of mirabilite for the treatment of acute pancreatitis. The use of topical mirabilite for post-ERCP pancreatitis prophylaxis may be better tolerated in pediatric patients by its external use nature and according toits safety profile in treatment of acute pancreatitis compared to rectal, oral, or intravenous applications.

In this study, we aim to assess the efficacy of mirabilite for post-ERCP pancreatitis prophylaxis by comparing the incidence of this condition between the intervention and control groups. Comparisons of the concentrations of inflammatory markers and the translocation of amine dioxide, diamine oxidase, and endotoxin between the two groups will enable a further demonstration of the probable mechanism of action of mirabilite via improved intestinal function consequent to inflammation suppression. This study will also enable an observation of the side effects of mirabilite. We expect that mirabilite will reduce the incidence of post-ERCP pancreatitis and suppress pain, reduce inflammation, and improve intestinal function in patients after ERCP. We expect that mirabilite will be confirmed as an effective and safe treatment option for post-ERCP pancreatitis prophylaxis in pediatric patients.

\section{Trial Status}

Protocol version number and date: SCMC 1708; Aug 20th, 2019.

The date recruitment began: Oct 22th, 2019.

The approximate date when recruitment will be completed: Oct 22th, 2021.

\section{Declarations}

Acknowledgements We thank Dr. Fu Li, the distinguished professor of Shanghai Shuguang Hospital Affiliated to Shanghai University of Traditional Chinese Medicine, for his review of the manuscript for optimal English language presentation. We also deliver our thanks to all the staff for their valuable support in participant recruitment. 
Funding This work is supported by grants from the Shanghai Municipal Health Commission of China (grant number 2018LP018, ZY (2018-2020)-FWTX-1105). The funders has no role in study design, data collection and analysis, decision to publish, or preparation of the manuscript.

Author contributions ZD, KY, JZ, CL, JJ, XL, GZ, BG and ZW conceived and designed the study and participated in logistical planning of the study. DZ drafted the initial version of the manuscript. $K Y, J Z, C L$, $\mathrm{JJ}, \mathrm{XL}$ and $\mathrm{GZ}$ acquired, analyzed, and interpreted the data. ZD, BG and ZW edited the manuscript. All authors agreed to be accountable for all aspects of the work.

Ethics approval and consent to participate The trial protocol has been approved by the Regional Ethical Review Board in Shanghai Children's Medical Center(SCMCIRB-K2019005). It will be monitored by Clinical Research Institute of Shanghai Jiao Tong University School of Medicine. The study will be conducted in accordance with the principles of the Declaration of Helsinki (2013 version). The study design will comply with the principles set out in the Good Clinical Practice (GCP) guidelines according to the theory that guides the appropriate use of Traditional Chinese medicine in clinical application. The study design is in accordance with the Standard Protocol Items: Recommendations for Interventional Trials (SPIRIT) guidelines (see Fig.1). Informed consent will be obtained from all participants prior to entry into the trial including the possible risks, benefits, and knowledge of the purpose of the trial. The Ethics Committee of the GPHCM will review the study progress at least once a year. Any formal amendment to the protocol required must be approved by the Ethics Committee of the Regional Ethical Review Board in Shanghai Children's Medical Center prior to implementation if modifications may impact on the conduct of the study, potential benefit of the patient, or may affect patient safety. For the RCT, trained researcher will introduce and discuss the trial information to interested patients, and a signed informed consent form will be obtained before each participant's enrollment. Participants will be free to withdraw at any time.

Conflict of Interest The authors declare no conflict of interest associated with this manuscript.

Consent for publication Not Applicable

\section{Author details}

${ }^{1}$ Department of Gastroenterology, Shanghai Children's Medical Center, Shanghai Jiao Tong University School of Medicine, Shanghai 200127, China. ${ }^{2}$ Department of Gastroenterology, Shanghai Shuguang Hospital Affiliated to Shanghai University of Traditional Chinese Medicine, Shanghai 200120, China. ${ }^{3}$ Clinical Research Institute, Shanghai Jiao Tong University School of Medicine, Shanghai 200025, China.

\section{References}

1. Jasmin Felux E, Sturm A, Busch, et al. ERCP in infants, children and adolescents is feasible and safe: results from a tertiary care center. United European Gastroenterology Journal. 2017;5:1024-29. 
2. Giefer MJ, Kozarek RA. Technical outcomes and complications of pediatric ERCP. Surg Endosc. 2015;29:3543-50.

3. Troendle DM, Abraham $\mathrm{O}$, Huang $\mathrm{R}$, et al. Factors associated with post-ERCP pancreatitis and the effect of pancreatic duct stenting in a pediatric population. Gastrointest Endosc. 2015;81:1408-16.

4. Danielle Usatin M, Fernandes IE, Allen, et al. Complications of Endoscopic Retrograde Cholangiopancreatography in Pediatric Patients; A Systematic Literature Review and Meta-Analysis. J Pediatr. 2016;179:160-5.

5. Giefer MJ, Kozarek RA. Technical outcomes and complications of pediatric ERCP. Surg Endosc. 2015;29:3543-50.

6. Coté GA, Sagi SV, Schmidt SE, et al. Early measures of hemoconcentration and inflammation are predictive of prolonged hospitalization from post- endoscopic retrograde cholangiopancreatography pancreatitis. Pancreas. 2013;42:850-4.

7. Tryliskyy Y, Bryce GJ. Post-ERCP pancreatitis: Pathophysiology, early identification and risk stratification. Adv Clin Exp Med. 2018;27:149-54.

8. Liu L, Li C, Huang Y, et al. Nonsteroidal Anti-inflammatory Drugs for Endoscopic Retrograde Cholangiopancreatography Postoperative Pancreatitis Prevention: a Systematic Review and Metaanalysis. J Gastrointest Surg. 2019;23:1991-2001.

9. Luo H, Zhao L, Leung J, et al. Routine pre-procedural rectal indometacin versus selective postprocedural rectal indometacin to prevent pancreatitis in patients undergoing endoscopic retrograde cholangiopancreatography: a multicentre, single-blinded, randomised controlled trial. Lancet. 2016;387:2293-301.

10. Zhang JW, Zhang GX, Chen HL, et al. Therapeutic effect of Qingyi decoction in severe acute pancreatitis-induced intestinal barrier injury. World J Gastroenterol. 2015;21:3537-46.

11. Zhao J, Tang W, Wang J, et al. Pharmacokinetic and pharmacodynamic studies of four major phytochemical components of Da-Cheng-Qi decoction to treat acute pancreatitis. J Pharmacol Sci. 2013;122:118-27.

12. Ma Y, Huang ZW, Xia Q, et al. Influence of integrated traditional Chinese and Western medicine therapy on serum resistin levels in patients with severe acute pancreatitis: a randomized controlled trial. Zhong Xi Yi Jiehe Xuebao. 2009;7:1134-8.

13. Wang YF, Zhang YH, Jiang RL. Early traditional Chinese medicine bundle therapy for the prevention of sepsis acute gastrointestinal injury in elderly patients with severe sepsis. Sci Rep. 2017;5:1-10.

14. Zhang MJ, Zhang GL, Yuan WB, et al. Treatment of abdominal compartment syndrome in severe acute pancreatitis patients with traditional Chinese medicine. World J Gastroenterol. 2008;14:357478.

15. Zhao J, Tang W, Wang J, et al. Pharmacokinetic and pharmacodynamic studies of four major phytochemical components of Da-Cheng-Qi decoction to treat acute pancreatitis. J Pharmacol Sci. 2013;122:118-27. 
16. Jang DK, Jung $\mathrm{CY}$, Kim KH, et al. Electroacupuncture for abdominal pain relief in patients with acute pancreatitis: study protocol for a randomized controlled trial. Trials. 2018;19:279-85.

17. Cotton PB, Lehman G, Vennes J, et al. Endoscopic sphincterotomy complications and their management: an attempt at consensus. Gastrointest Endosc. 1991;37:383-93.

\section{Figures}

\section{Participant recruitment}

Screening and assessment for eligibility
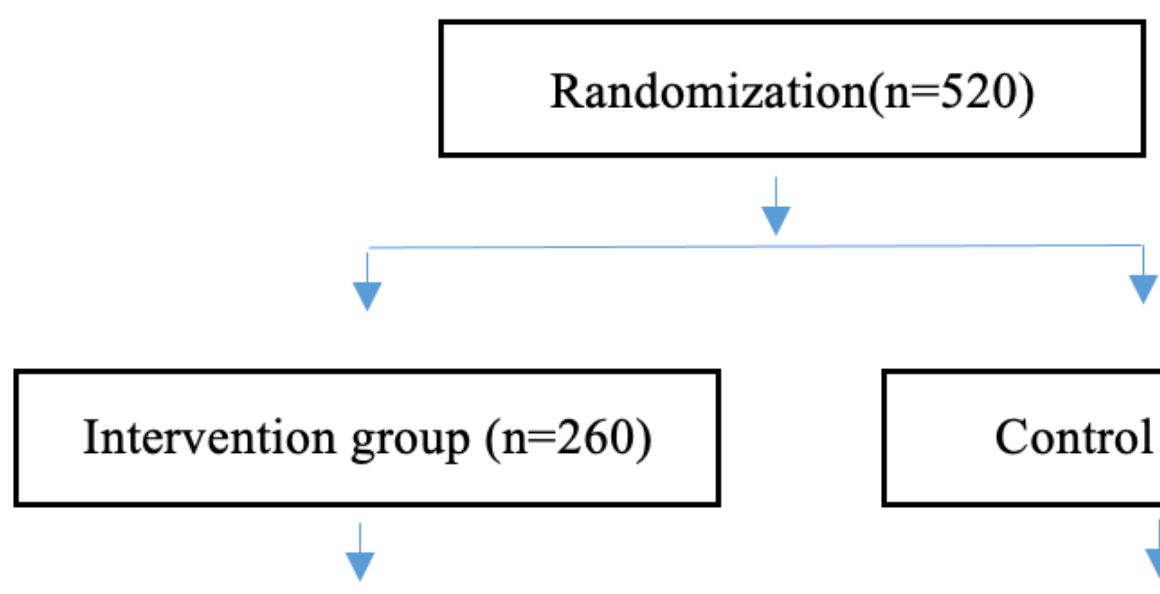

Control group $(\mathrm{n}=260)$

External use of Mirabilite

No any additional intervention

Outcome measurement

Data collection and statistic analysis 
Figure 1

Study design flow chart

\begin{tabular}{|c|c|c|c|c|c|c|}
\hline \multirow[b]{2}{*}{ TIMEPOINT } & \multirow{2}{*}{$\begin{array}{l}\text { Enrolment } \\
\text { Pre } \\
\end{array}$} & \multirow{2}{*}{$\begin{array}{l}\text { Allocation } \\
0 \mathrm{D}\end{array}$} & \multicolumn{3}{|c|}{ Post-allocation } & \multirow{2}{*}{$\begin{array}{l}\text { Close- } \\
\text { out } \\
1 \mathrm{M} \\
\end{array}$} \\
\hline & & & $1 \mathrm{D}$ & $2 \mathrm{D}$ & $3 \mathrm{D}$ & \\
\hline \multicolumn{7}{|l|}{ Research activities } \\
\hline Enrollmemt & $\mathrm{x}$ & & & & & \\
\hline Eligibility screen & $\mathrm{x}$ & & & & & \\
\hline Sign informed comment & $\mathbf{x}$ & & & & & \\
\hline Randomization & $\mathrm{x}$ & & & & & \\
\hline \multicolumn{7}{|l|}{ Intervention activities } \\
\hline $\begin{array}{l}\text { Intervention group } \\
\text { External use of Mirabilite } \\
\text { Control group } \\
\text { No any additional intervention } \\
\text { Date collection }\end{array}$ & & $\mathrm{x}$ & & & & \\
\hline Baseline assessments & $\mathrm{x}$ & & & & & \\
\hline Socio-demographics & $\mathrm{x}$ & & & & & \\
\hline Anthropometric variables & $\mathrm{x}$ & & & & & \\
\hline Medical history & $\mathrm{x}$ & & & & & \\
\hline \multicolumn{7}{|l|}{ Outcome assessments } \\
\hline Abdominal pain scores & & $\mathrm{x}$ & & & & \\
\hline amylase levels & & $\mathrm{x}$ & & & & \\
\hline $\begin{array}{ll}\text { upper } & \text { abdominal } \\
\text { ultrasonography }\end{array}$ & & $\mathrm{x}$ & & & & \\
\hline TNF- $\alpha$, IL-6, IL-8 & & $\mathrm{x}$ & $\mathrm{x}$ & $\mathrm{x}$ & $\mathrm{x}$ & \\
\hline $\begin{array}{l}\text { diamine oxidase, blood D-lac, } \\
\text { endotoxin } \\
\text { Safety }\end{array}$ & & $\mathrm{x}$ & $\mathrm{x}$ & $\mathrm{x}$ & $\mathrm{x}$ & \\
\hline Adverse events & & $\mathbf{x}$ & $\mathrm{x}$ & $\mathrm{x}$ & $\mathrm{x}$ & $\mathrm{x}$ \\
\hline
\end{tabular}

D day, $\mathrm{M}$ month

Figure 2

Treatment schedule and outcome measures. 


\section{Supplementary Files}

This is a list of supplementary files associated with this preprint. Click to download.

- SPIRITChecklist13.doc

- InformedConsentforguardian.docx

- InformedConsentforchildren.docx 